\title{
Evaluating the accuracy of emergency nurses in correct triage using emergency severity index triage in Sina hospital of Tabriz: a cross-sectional analysis
}

\author{
Farzad Rahmani', Pegah Sepehri Majd ${ }^{2}$, Haniyeh Ebrahimi Bakhtavar ${ }^{3}$, Farnaz Rahmani ${ }^{*}$ \\ 'Road Traffic Injury Research Center, Tabriz University of Medical Sciences, Tabriz, Iran \\ 2Department of Internal Medicine, Tabriz branch, Islamic Azad University, Tabriz, Iran \\ ${ }^{3}$ Emergency Medicine Research Team, Tabriz University of Medical Sciences, Tabriz, Iran \\ ${ }^{4}$ Student Research Committee, School of Nursing and Midwifery, Tabriz University of Medical Sciences, Tabriz, Iran
}

\author{
Received: 3 March 2017 \\ Accepted: 23 May 2017 \\ Published online: 1 June 2017 \\ *Corresponding author: Farnaz \\ Rahmani, Emergency Medicine \\ Department, Sina medical research \\ and training hospital, Tabriz \\ University of Medical Sciences, \\ Tabriz, Iran. Tel: +989143002439; \\ Fax: +98 3 [41] 4796969; \\ Email: \\ farnazrahmani58@gmail.com \\ Competing interests: None. \\ Funding information: None. \\ Citation: Rahmani F, Sepehri Majd \\ P, Ebrahimi Bakhtavar H, Rahmani \\ F. Evaluating the accuracy of \\ emergency nurses in correct triage \\ using emergency severity index \\ triage in Sina hospital of Tabriz: a \\ cross-sectional analysis. Journal of \\ Emergency Practice and Trauma \\ 2018; 4(1): 9-13. doi: 10.15171/ \\ jept.2017.19.
}

\begin{abstract}
Objective: Triage refers to the evaluation and categorization of patients for better management to deliver services. A good triage system should be able to accurately specify the patients who require urgent care. Thus, this study aimed to evaluate the accuracy of emergency nurses in correct triage using Emergency Severity Index (ESI) triage.

Methods: In this descriptive cross-sectional study 750 patients, who referred to the emergency department (ED) of Sina teaching hospital, participated from 23 July to 22 August in 2015. Participants were selected using convenience sampling method. The patients' triage level was determined by physicians and nurses separately and the results were compared. To define the level of agreement between two groups (inter-rater agreement), the kappa index was evaluated. Data were analyzed using SPSS version 17. Results: Among 750 patients, 577 patients (76.9\%) were classified in correct triage group, 90 patients (12\%) in undertriage group and 83 patients (11.1\%) in overtriage group. Kappa agreement coefficient between the ESI triage level of physicians and nurses was 0.659 (95\% Cl: 0.646-0.667). There was statistically significant difference between the triage level of physicians and nurses $(P<0.001)$.

Conclusion: This study showed that there was moderate agreement between the triages of physicians and nurses. It seems that improving nurses' skills and knowledge might increase the accuracy of emergency nurses in patients' correct triage. Therefore, planning programs based on training correct triage for emergency nurses is strongly suggested. Keywords: Emergency department, Triage, Nurses, Physicians
\end{abstract}

\section{Introduction}

In emergency rooms (ERs), seconds and minutes are important for the patient because this time may determine the distance between the time of death, serious disability or return to life (1). Emergency circumstances are dangerous situations where the physical or psychological conditions of people suddenly deteriorate and immediate needs and appropriate measures are crucial (2). Using triage system is a proper approach to prioritize medical needs for patients in the emergency departments (EDs) (3).

A good triage system should be able to accurately identify patients in need of emergency care, guiding them to the right path with fast access to diagnostic and therapeutic treatment. An incorrect triage leads to a waste of resources, delays in patients' treatment, discontent and undesirable consequences (4). One of these triage systems is Emergency Severity Index (ESI) system, which in 1999, its first edition was developed by Wuerz and Eitel, and four editions have been conducted so far (1). This system of triage has five levels and the triage nurse classifies the patient based on two criteria of disease severity and required facilities (1-5).

In patients' triage, internal factors including nurses' skills, experience, knowledge, personal characteristics, work environment, increased work, and patients' evaluation are important (2). Findings of a study showed that there was no significant difference between nurses and doctors in terms of triaging patients (6). In another study, it was concluded that undertriage in elderly patients was still a problem (7). If the triage nurse does not observe the 
principles of triage in classification of patient, a mistake as overtriage or undertriage will happen which can be dangerous for patients who are at this level and need lifesaving measures (1).

Undertriage refers to situations where the triage nurse estimates the patient acuity less than the actual level and causes delay in patient's treatment. Overtriage refers to situations where the triage nurse estimates the patient acuity higher than the current and makes the physicians visit the patient instead of the really ill patient. In terms of the complications for the patient, undertriage is more dangerous (6-9). No reasonable amount is defined for these two states but the goal is to reach undertriage below $10 \%$ (7). Neglect of risky situations and lack of proper interpretation of vital signs are the main reasons for undertriage. Undertriage in ED can lead to side effects for hospitalized patients during their stay in hospital, and on the other hand, overtriage mainly creates problems in the consumption of resources and diverting medical staff from other important activities in the hospital (3).

Sina teaching hospital is a general hospital affiliated to the Tabriz University of Medical Sciences having internal, poisoning, surgery, urology, burns, dermatology, orthopedics wards and four intensive care units. ED of this center is a general ward and each year approximately 40000 patients are admitted with different complaints (10). Every month, about 3000-3500 patients are admitted to this ED. So, proper diagnosis of patients needed triage is necessary. The aim of this study was to $(a)$ evaluate the accuracy of emergency nurses in patients' correct triage using ESI and $(b)$ determine the number of undertriaged and overtriaged patients.

\section{Methods}

This descriptive cross-sectional study was conducted on 750 patients who referred to the ED of Sina teaching hospital from 23 July to 22 August in 2015.

To calculate the sample size using Morgan table and considering $\alpha=0.05$ and reliability of $99 \%$, sample size was estimated 650 patients. Considering the possible 15\% loss of sample, 750 patients were chosen to increase the accuracy of the study.

Inclusion criteria included patients admitted to the ED of Sina hospital over a period of one month (23 July-22 August) in 2015. Exclusion criteria included incomplete records of patients. All ethical requirements were met in the study and information of all patients remained confidential.

To collect data, we used two questionnaires. The first questionnaire included demographic information for both patients (age, sex, date, time of patient's referral and cause of admission) and nurses (age and gender of the triage nurse, work experience based on years of working in the ER, triage level by the nurse). In addition, triage level was determined by the physician in re-triage in the second part of the questionnaire. Hospitalization, discharging from ED and mortality were considered as the outcomes. The second questionnaire, ESI, is a five-level ED triage algorithm that provides clinically relevant stratification of patients into five groups from 1 (most urgent) to 5 (least urgent) on the basis of acuity and resource needs. The ESI is unique among triage tools, by including both acuity and resource needs in the system of categorizing ED patients $(11,12)$. This standard and national index is used by emergency team in all EDs of hospitals in Iran. Tanabe et al confirmed the high reliability of five-level triage, and reported a high reliability for it $($ Kappa $=0.89)(13)$. Some studies reported that ESI is a valid and reliable method for better allocation of resources and better classification of patients to get appropriate treatment $(14,15)$.

In the first moment of the patient's admission to ER, demographic data (age, sex, date, time of patient's referral and cause of admission) were obtained. The initial assessment of the triage nurse was registered in the national standard triage checklist and the level of patient's triage was determined. Finally the patient was admitted. After visiting the patient, the physician compared the triage level determined by himself with the triage level assessed by the nurse, and in case of any differences, the physician in charge conducted a re-triage. Two emergency medicine specialists (faculty members) of Sina hospital ED had cooperation to determine the exact level of triage and stated their opinions on the triage and re-triage levels. If there were any discrepancies, a third person (emergency medicine specialist or a faculty member) was negotiated. Data were analyzed using SPSS statistical software (model number: 17.0.1, SPSS Inc, Chicago). Data distribution normality was evaluated using Kolmogorov-Smirnov test. Descriptive tests included mean \pm standard deviation (SD), frequency and percentage. Chi-square test was used to compare qualitative data. Kappa agreement coefficient was used to determine the agreement between triage levels of the nurse and physician. A $P$ value of $<0.05$ was considered statistically significant.

\section{Results}

The majority of patients (52.8\%) were male. Most of them (79.1\%) were married. Mean \pm SD of the patients' age was $41.79 \pm 20.64$ years. Most patients $(96.4 \%)$ were referred to the ER by their family.

$37.1 \%$ and $74.8 \%$ of patients were admitted to the ER in the night shift and during the working days respectively. $67.6 \%$ of patients were discharged.

Regarding the accuracy of triage nurse, 577 patients (76.9\%) experienced correct triage, 90 patients (12\%) were undertriaged and 83 patients (11.1\%) were overtriaged. Table 1 compares nurse's triage and the physician's final triage. There was statistically significant difference between the triage carried out by physicians and nurses $(P$ $<0.001)$. Kappa coefficient agreement between the nurse's triage and the final triage by the physician was 0.659 (95\% CI: 0.646-0.667), which showed an average level of agreement between the two groups.

The majority of patients $(86.8 \%)$ were triaged by female nurses and most of them (78.5\%) experienced correct triage. But $11.1 \%$ experienced overtriage and $10.4 \%$ 
Table 1. Comparison of nurse's triage and physician's re-triage

\begin{tabular}{|c|c|c|c|c|c|}
\hline \multirow{2}{*}{ ESI level (Nurse) } & \multicolumn{5}{|c|}{ ESI level(Physician) } \\
\hline & ESI level 1 & ESI level 2 & ESI level 3 & ESI level 4 & ESI level 5 \\
\hline ESI level 1 & 13 & 1 & 0 & 0 & 0 \\
\hline ESI level 2 & 12 & 127 & 9 & 2 & 1 \\
\hline ESI level 3 & 2 & 47 & 291 & 27 & 15 \\
\hline ESI level 4 & 0 & 5 & 20 & 134 & 29 \\
\hline ESI level 5 & 0 & 0 & 1 & 3 & 12 \\
\hline
\end{tabular}

experienced undertriage. On the other hand, $66.7 \%$ of patients were triaged by male nurses experienced correct triage. In this regard, $11.1 \%$ experienced overtriage and 22.2\% experienced undertriage. There was statistically significant difference in the accuracy of triage by male and female nurses in the $\operatorname{ED}(P=0.003)$, indicating that the triages by female nurses were more correct. The average working time of nurses in the ED was $2.53 \pm 1.95$ years (minimum: one year and maximum: 8 years, median: 2 years).

The frequency of correct and incorrect triage based on the years of work in the ED is shown in Table 2. As the table shows, many of the patients identified as undertriage were categorized by nurses with one year of work experience. The ideal triages were conducted by nurses with six years of work experience.

Table 3 shows the triage error in various work shifts, working days and holidays. As the table shows, there was a significant difference in triage error between different shifts $(P=0.039)$; undertriage was higher at night, while overtriage was higher in the morning. Based on working days and holidays, there was no statistical significant difference in the triage error $(P=0.077)$.

\section{Discussion}

According to the findings of this study, there was moderate agreement between the triages of physicians and nurses.

Table 2. The relationship between nurses' experience and correct triage

\begin{tabular}{lccc}
\hline \multirow{2}{*}{$\begin{array}{l}\text { Occupational } \\
\text { history }\end{array}$} & \multicolumn{3}{c}{ Triage accuracy } \\
\cline { 2 - 4 } & True level & Undertriage & Overtriage \\
\hline 1 & $146(68.5 \%)$ & $38(17.8 \%)$ & $29(13.6 \%)$ \\
\hline 2 & $281(81.7 \%)$ & $26(7.6 \%)$ & $37(10.8 \%)$ \\
\hline 3 & $74(76.3 \%)$ & $11(11.3 \%)$ & $12(12.4 \%)$ \\
\hline 6 & $14(82.4 \%)$ & $2(11.8 \%)$ & $1(5.9 \%)$ \\
\hline 8 & $26(81.3 \%)$ & $4(12.5 \%)$ & $2(6.3 \%)$ \\
\hline
\end{tabular}

According to Esmailian et al, the triage of nurse and doctor overlapped in $90 \%$ of cases and weighted Kappa was $94 \%$ (6). But in the study of Goransson and von Rosen, only $58 \%$ of nurses' triage was acceptable (16). Abbasi et al also determined that the accuracy of nurses' triage was low (17). On the contrary, Worster et al found a high accuracy for nurses' triage (18). Quick and accurate triage of patients in the ED is the key to successful performance and in the case of selecting improper level of triage based on misreading or ignoring patients' variables and triage criteria, nurses will face triage errors. Undertriage and overtriage are important errors in the triage by nurses (3). To reduce overtriage, it is recommended to use a simple and functional triage system (11). EDs' managers need accurate information to improve the quality of triage and reduce errors to ensure correct implementation of triage and identify nurses' needs and gaps in training (3).

The triage done by a nurse has high credibility like the triage conducted by the doctor (4). It should also be noted that triage is a practical work, but without adequate knowledge, we can never expect a proper function $(4,12)$. Buschhorn et al found that the agreement between the triage of trained nurses and pre-hospital emergency staff was moderate (19).

According to Kariman et al, the sensitivity of ESI in identification of old patients who should receive lifepreserving interventions was low (5). As Platts-Mills et al reported, this can be related to the fact that nurses did not triage patients directly; they only referred to the patient's past medical history (15).

Although the majority of patients experienced correct triage, but $12 \%$ experienced undertriage and $11.1 \%$ experienced overtriage. The triage error rate was higher than our considered rate (10\%) which is consistent with the finding of Källberg et al (20). Furthermore, the most common contributing factor to triage error was human error, in line with the finding of Källberg et al (20).

According to the findings, with increasing the years of work and age in ED, triage error rate in emergency nurses

Table 3. Triage error in various work shifts, and working days and holidays

\begin{tabular}{|c|c|c|c|c|c|}
\hline \multirow{2}{*}{ Time } & & \multicolumn{3}{|c|}{ Triage accuracy } & \multirow[b]{2}{*}{ P value } \\
\hline & & True level & Undertriage & Overtriage & \\
\hline \multirow{3}{*}{ Shift } & Morning & $182(80.2 \%)$ & $15(6.6 \%)$ & 30 (13.2\%) & \multirow{3}{*}{0.039} \\
\hline & Evening & $188(76.7 \%)$ & 34 (13.9\%) & $23(9.4 \%)$ & \\
\hline & Night & 207 (74.5\%) & 41 (14.7\%) & $30(10.8 \%)$ & \\
\hline \multirow{2}{*}{ Day } & Working Day & $433(77.2 \%)$ & $73(13 \%)$ & $55(9.8 \%)$ & \multirow{2}{*}{0.077} \\
\hline & Holiday & 144 (76.2\%) & $17(9 \%)$ & $28(14.8 \%)$ & \\
\hline
\end{tabular}


is reduced. This is consistent with other studies (21-25). Emergency nurses Association of America states that triage nurses must have at least 6 months of experience in emergency (2). But other studies have found that there is no direct relationship between the work experience and the scores obtained in triage knowledge questionnaire $(21,26)$. Considine et al reported that factual knowledge may be more important than years in emergency nursing or triage experience in triage decision accuracy (23).

Based on the findings, undertriage was higher at night, while overtriage was higher in the morning. This is in line with Göransson et al study (25). A possible explanation is that triage nurses have high accuracy in patients' triage in the morning because they pay attention to everything. While at night, undertriage is higher probably due to fatigue and high level of admitted patients. This is consistent with other studies $(27,28)$.

One of the best interventions to reduce the triage error rate is to provide necessary triage trainings to staff members of the triage unit and continuous monitoring of their performance (3). Triage training affects nurses' knowledge and performance in the ED (12). Problem-based ESI triage training can significantly reduce the duration of triage by triage nurses and improve their performance (1).

\section{Conclusion}

The present study showed that there was moderate agreement between nurses and physicians in triage level. It can be concluded that many triage education programs are needed to improve accurate triage decision making in nurses. According to the large number of referred patients in early hours of the night and high level of undertriage in the night shifts, it is recommended to have two nurses in the ED at these times to prevent errors.

This study has several limitations. First, there were a limited number of experienced nurses in the ED due to the lack of human resources. Therefore, this may affect results and increase the rate of triage errors. Next, the impact of triage error on the quality of services to patients and their outcome was not assessed. Further studies are recommended to assess the impact of triage error on the patients' outcome.

\section{Ethical issues}

This study was approved by the ethics committee of Tabriz Islamic Azad University.

\section{Authors' contributions}

All authors have read and approved the manuscript. FR, PSM, and HEB performed the data collection, writing, critical revision and drafting of the manuscript. FR undertook the major parts of the study design and performed the statistical analysis, data analysis and data interpretation.

\section{References}

1. Khatiban M, Khazaei A, Karampourian A, Soltanian A, Kimiaie Asadi H, Salimi R, et al. The effects of the
Emergency Severity Index triage education via problem based learning on the triage nurses' performance and the patients' length of stay in the Emergency Department. Journal of Clinical Research in Paramedical Sciences 2014; 3(2): 63-74. [in Persian].

2. Tabatabai A, Mohammadnejad E, Salari A. Nursing students' awareness of triage in the emergency ward. Hakim Seyed Esmail Jorjani Journal 2014; 1(1): 30-4. [In Persian].

3. Kamrani F, Ghaemipour F, Nikravan M, Alavi Majd H. Prevalence of miss triage and outcomes under triage of patients in emergency department. Journal of Health Promotion Management 2013; 2(3): 17-23. [in Persian]

4. Gilboy N, Tanabe P, Travers D, Rosenau AM. Emergency Severity Index (ESI): A Triage Tool for Emergency Department Care. 4th ed. Rockville, MD: AHRQ Publication; 2012.

5. Kariman H, Joorabian J, Shahrami A, Alimohammadi H, Noori Z, Safari S. Accuracy of emergency severity index of triage in Imam Hossein hospital-Tehran, Iran (2011). Journal of Gorgan University of Medical Sciences 2013; 15(1): 115-20. [in Persian]

6. Esmailian M, Zamani M, Azadi F, Ghasemi F. Interrater agreement of emergency nurses and physicians in Emergency Severity Index (ESI) Triage. Emerg (Tehran) 2014; 2(4): 158-61. doi: 10.22037/emergency.v2i4.6449.

7. Grossmann FF, Zumbrunn T, Ciprian S, Stephan FP, Woy $\mathrm{N}$, Bingisser $\mathrm{R}$, et al. Undertriage in older emergency department patients-tilting against windmills? PLoS One 2014; 9(8): e106203. doi: 10.1371/journal.pone.0106203.

8. Newgard CD, Staudenmayer K, Hsia RY, Mann NC, Bulger EM, Holmes JF, et al. The cost of overtriage: more than one-third of low-risk injured patients were taken to major trauma centers. Health Aff (Millwood) 2013; 32(9): 1591-9. doi: 10.1377/hlthaff.2012.1142.

9. Faul M, Wald MM, Sullivent EE, Sasser SM, Kapil V, Lerner EB, et al. Large cost savings realized from the 2006 Field Triage Guideline: reduction in overtriage in U.S. trauma centers. Prehosp Emerg Care 2012; 16(2): 222-9. doi: 10.3109/10903127.2011.615013.

10. Eshghi M, Rahmani F, Derakhti B, Abdollahi F, Tajoddini S. Patient satisfaction in the emergency department: a case of Sina hospital in Tabriz. Journal of Emergency Practice and Trauma 2016; 2(1): 16-20. doi: 10.15171/jept.2015.06.

11. Lehmann R, Brounts L, Lesperance K, Eckert M, Casey L, Beekley A, et al. A simplified set of trauma triage criteria to safely reduce overtriage: a prospective study. Arch Surg 2009; 144(9): 853-8. doi: 10.1001/archsurg.2009.153.

12. Kalantarimeibidi M, Yadollahi A, Esfandiari S. The effect of education on the knowledge and practice of emergency department's nurses regarding the patients' triage. Iran J Emerg Med 2014; 1(1):40-44.

13. Tanabe P, Gimbel R, Yarnold PR, Kyriacou DN, Adams JG. Reliability and validity of scores on The Emergency SeveritynIndex version 3. Acad Emerg Med 2004; 11(1): 59-65. doi: 10.1197/S1069-6563(03)00585-2.

14. Wuerz RC, Milne LW, Eitel DR, Travers D, Gilboy N. Reliability and validity of a new five-level triage instrument. Acad Emerg Med 2000; 7(3): 236-42. doi:10.1111/j.1553-2712.2000.tb01066.x.

15. Platts-Mills TF, Travers D, Biese K, McCall B, Kizer S, LaMantia M, et al. Accuracy of the Emergency Severity Index triage instrument for identifying elder emergency department patients receiving an immediate life-saving 
intervention. Acad Emerg Med 2010; 17(3): 238-43. doi: 10.1111/j.1553-2712.2010.00670.x.

16. Goransson KE, von Rosen A. Interrater agreement: a comparison between two emergency department triage scales. Eur J Emerg Med 2011; 18(2): 68-72. doi: 10.1097/ MEJ.0b013e32833ce4eb.

17. Abbasi E, Nosrati A, Nabipour I, Emami SR. Assessment of the level of knowledge of physicians in Bushehr province about preparedness and response for nuclear emergency. Iranian Sought Medical Journal 2005; 7(2): 183-89. [in Persian]

18. Worster A, Sardo A, Eva K, Fernandes CM, Upadhye S. Triage tool inter-rater reliability: a comparison of live versus paper case scenarios. J Emerg Nurs 2007; 33(4): 31923. doi: 10.1016/j.jen.2006.12.016.

19. Buschhorn HM, Strout TD, Sholl JM, Baumann MR. Emergency medical services triage using the emergency severity index: is it reliable and valid? J Emerg Nurs 2013; 39(5): e55-63. doi: 10.1016/j.jen.2011.11.003.

20. Källberg AS, Göransson KE, Östergren J, Florin J, Ehrenberg A. Medical errors and complaints in emergency department care in Sweden as reported by care providers, healthcare staff, and patients - a national review. Eur J Emerg Med 2013; 20(1): 33-8. doi: 10.1097/MEJ.0b013e32834fe917.

21. Mirhaghi AH, Roudbari M. Survey on knowledge level of the nurses about hospital triage. Iranian Journal of Critical Care Nursing 2011; 3(4): 167-74. [in Persian]

22. Sedaghat S, Aghababaeian H, Taheri N, Sadeghi Moghaddam A, Maniey M, Araghi Ahvazi L. Study on the level of knowledge and performance of North Khuzestan medical emergency 115 personnel on prehospital triage. Iran J Crit Care Nurs 2012; 5(2): 103-8.

23. Considine J, Botti M, Thomas S. Do knowledge and experience have specific roles in triage decision-making? Acad Emerg Med 2007; 14(8): 722-6.

24. Hitchcock R. Speeding up the ED care process. Three hospital organizations mitigate overcrowding by improving patient flow, processes and documentation. Health Management Technology 2012; 33(12): 6-8.

25. Göransson KE, Ehrenberg A, Marklund B, Ehnfors M. Emergency department triage: is there a link between nurses' personal characteristics and accuracy in triage decisions? Accid Emerg Nurs 2006; 14(2): 83-8. doi: 10.1016/j.aaen.2005.12.001

26. Hedayati H, Mogharrab M, Moasheri N, Sharifzadeh G. Studying of BUMS' students' knowledge about hospital triage in 2011. Modern Care Journal 2013; 9(3): 237- 44. [In Persian]

27. Dadashzadeh A, Abdollahzadeh F, Shams Vahdati S, Lotfi M, Ghojazadeh M, Bagheri Mehmandousti S. Causes of delay in patient triage in the emergency departments of Tabriz hospitals. Turk J Emerg Med 2011; 11(3): 95-8. doi: 10.5505/1304.7361.2011.59354.

28. Azhough R, Shams Vahdati S, Faraji F, Faraji M, Ghorbanian M, Ramouz A, Tajoddini S. One-day triage course for nurses, it is essential. Journal of Emergency Practice and Trauma 2015; 1(2): 52-5 